\title{
Tomar partido: práctica artística y visibilización de conflictos, el caso del proyecto "Nos Jardins".
}

\author{
Anaïs Florin \\ Universitat Politènica de València, anaisflorin@gmail.com
}

\begin{abstract}
The Nos Jardins project (i.e. Our community garden) was developed between May and June of 2019 within the Bien Urbain, Art dans et avec l'espace public annual public festival in Bensançon (France). Nos Jardins addresses the problem in the Les Vaîtes neighborhood. Where since 2005 exist an urban development project promoted by the municipal government that intends to wipe out a border area between the countryside and the city where historic worker community garden are located.

The Nos Jardins project consisted of a total of 26 illegal interventions in the public space in several neighborhoods of Besançon. All interventions were carried out on advertising media (muppies, canopies and fences). The project aimed to generate an artistic intervention device in the public space that allowed to give visibility to the conflict, and to be easily appropriated in its dissemination by different audiences.
\end{abstract}

Keywords: Context, activism, fight, territory, city, intervention

\begin{abstract}
Resumen
El proyecto Nos jardins (Nuestros huertos) fue desarrollado entre el mes de mayo y el mes de junio de 2019 dentro del marco del festival anual Bien Urbain, Art dans et avec l'espace public en Bensançon en Francia. Nos jardins aborda la problemática existente en el barrio bisontino Les Vaîtes donde existe desde 2005 un proyecto urbanístico promovido por la municipalidad que pretende arrasar con una zona limítrofe entre el campo y la ciudad en la que se encuentran unos huertos obreros históricos. El proyecto Nos jardins consistió en un total de 26 intervenciones ilegales en el espacio público en varios barrios de Besançon, todas realizadas en soportes publicitarios (muppies, marquesinas y vallas). El proyecto tenía como objetivo generar un dispositivo de intervención artística en el espacio público que permitiera dar visibilidad al conflicto de fácil apropiación en su difusión por parte de los diferentes públicos.
\end{abstract}

Palabras clave: Contexto, activismo, lucha, territorio, ciudad, intervención 


\section{Introducción}

El proyecto que expondré a continuación, Nos jardins (Nuestros huertos) fue desarrollado entre el mes de mayo y el mes de junio de 2019 dentro del marco del festival anual Bien Urbain, Art dans et avec l'espace public ${ }^{l}$ en Bensançon en Francia. Nos jardins aborda la problemática existente en el barrio bisontino "Les Vaîtes" donde existe desde 2005 un proyecto urbanístico promovido por la municipalidad que pretende arrasar con una zona limítrofe entre el campo y la ciudad en la que se encuentran unos huertos obreros históricos.

El proyecto Nos jardins consistió en un total de 26 intervenciones ilegales en el espacio público en varios barrios de Besançon, todas realizadas en soportes publicitarios (muppies, marquesinas y vallas). El proyecto tenía como objetivo generar un dispositivo de intervención artística en el espacio público que permitiera dar visibilidad al conflicto de fácil apropiación en su difusión por parte de los diferentes públicos.

He tomado la decisión de redactar este texto en primera persona del singular, saliéndome al menos esta vez del "nosotros" académico, difuso y educado, como muestra de cariño y compromiso por las experiencias y los aportes vitales que suponen los proyectos que compartes con otras personas. Se trata aquí de contar una experiencia encarnada que da lugar a un conocimiento encarnado, aunque siempre me acompañen, para darme luz en el camino, las palabras de aquellos y aquellas que ya pensaron sobre aspectos de la realidad que me interpelan.

\section{Les Vaîtes, cultivar los posibles}

Les Vaîtes es un barrio situado en una zona limítrofe entre el campo y la ciudad al este de Besançon (en Francia) en la que se encuentran unos huertos obreros históricos. En el origen, Les Vaîtes era una zona de explotación agraria en la que convivían explotaciones de gran tamaño gestionadas por familias terratenientes y explotaciones más pequeñas, parcelas arrendadas a agricultores particulares. Con el tiempo, casi la totalidad de las parcelas fueron arrendadas a particulares, en su mayoría familias obreras que cultivaban para el autoconsumo y que pagaban un precio simbólico por el uso y cuidado de la tierra. Al margen de la herencia agraria, la relevancia de Les Vaîtes en términos medioambientales tiene que ver con todo un ecosistema local, compuesto por árboles centenarios, zonas húmedas y diversas especies vegetales y animales, de las cuales algunas están protegidas.

En 2005, el ayuntamiento empezó a promover un proyecto urbanístico nuevo para la creación de un supuesto ecobarrio ${ }^{2}$ que conllevó la expropiación de una gran cantidad de parcelas agrícolas que fueron recalificadas a posteriori como suelo urbanizable. El proyecto implicaba la destrucción casi total de los huertos y de los espacios naturales mantenidos hasta la fecha por una comunidad de personas fuerte, espontánea y auto-organizada. Desde el comienzo, el proyecto fue rechazado por la comunidad local generando agrupaciones de vecinos y vecinas en contra de su implantación. En 2016, el movimiento de La Nuit Debout de Besançon apadrinó la causa y nombró el barrio Les Vaîtes Z.A.C. (Zona a cultivar) ${ }^{3}$.

\footnotetext{
${ }^{1}$ ASSOCIATION JUSTE ICI.Bien Urbain, Art dans et avec l'espace public <https://bien-urbain.fr > [Consulta: 30 de agosto de 2019]

${ }^{2}$ Para más información sobre el proyecto del ecobarrio: TERRITOIRE 25. L'écoquartier Les Vaîtes < $\underline{\text { http://www.les-vaites.fr/> [Con- }}$ sulta: 30 de agosto de 2019]

${ }^{3}$ Z.A.C. (Zones à Cultiver, zonas a cultivar) es un juego de palabra, originalmente las siglas de Z.A.C. se refieren a una Zone d'Aménagement Concerté (zonas de ordenación concertada).
} 
Después de varios años de silencio, en octubre de 2018 la municipalidad anunció el comienzo de las obras y una parte de las tierras en cultivo fueron arrasadas. A raíz de la retoma del proyecto se crea una nueva asociación, la asociación Les jardins des Vaîtes ${ }^{4}$ que consiguió, después de realizar varias acciones y de tomar la vía legal, paralizar las obras en febrero 2019 hasta nueva orden ${ }^{5}$.

A finales de 2018, el festival Bien Urbain, Art dans et avec l'espace public me invita, junto a varias artistas, a proponer una intervención en el espacio público de Besançon para su celebración en junio de 2019. Para el desarrollo del proyecto, nos dan vía libre en cuanto a temática y nos ofrecen recibirnos un mes entero. En un primer de contacto en febrero de 2019 nos enseñan Besançon y nos llevan a ver la zona de Les Vaîtes. Rápidamente tomé la decisión de indagar sobre la problemática, investigar todo lo que me ofrece la Web sobre el tema y generar unos primeros contactos con personas afectadas por la destrucción de los huertos en la distancia. Esos primeros contactos formaban parte de la asociación Les jardins des Vaîtes, en su mayoría militantes y ecologistas, fueron cruciales para entender la problemática en términos de contexto político y medioambiental.

Así, en mayo de 2019, sin tener nada muy claro en cuanto al proyecto a realizar, llegué a Besançon para un mes. Al llegar, pude conocer en vivo a las personas que me habían guiado en la distancia. Con ellas visité por primera vez los huertos. A raíz de estos primeros paseos, empecé a caminar y pasear sola, todos los días, por la mañana y por la tarde durante un mes. Durante un mes estuve paseando, conversando y recolectando anécdotas y sentires de personas que cultivan las tierras de la zona. Los perfiles eran ricos y dispares, y se entrelazaban con harmonía en torno al cuidado de las parcelas. Algunas personas eran hijas de campesinas y campesinos, otras migrantes, ecologistas, activistas, vecinas, no tan vecinas, jóvenes y jubiladas. La organización de los huertos no funcionaba con asambleas ni reuniones, no existía una voluntad enunciada de autonomía por la colectividad, pero así sucedía. Si una persona quería una parcela, iba allí, preguntaba y se le indicaba cuáles estaban libres en ese momento. Lo mismo sucedía con los recursos, por ejemplo, con el uso del agua. La totalidad de los huertos se regaba con agua de lluvia y varias pequeñas charcas naturales, conllevando, además, un uso responsable del agua en todas las parcelas.
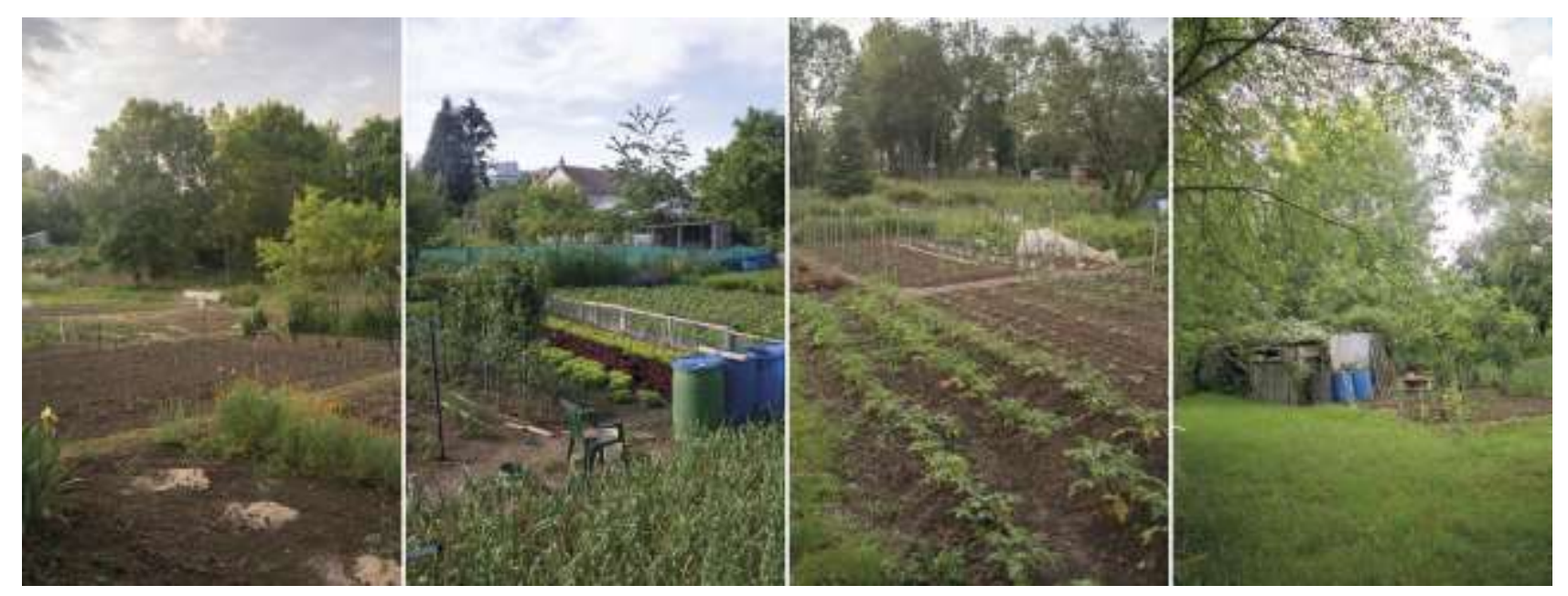

Fig. 1: Les Vaîtes

Fotografía: Anaïs Florin.

\footnotetext{
${ }^{4}$ Toda la información sobre las acciones llevadas a cabo por la asociación aquí: AsSOCIATION LES JARDINS DES VAîTES. Les jardins des Vaîtes <https://www.facebook.com/jardinsdesvaites/> [Consulta: 30 de agosto de 2019]

${ }^{5}$ COURAGeOT, S. (2019) "Besançon : Les travaux du futur éco-quartier des Vaîtes suspendus par le tribunal administratif.", 5 de mayo.

<https://france3-regions.francetvinfo.fr/bourgogne-franche-comte/doubs/besancon/besancon-travaux-du-futur-eco-quartier-vaitessuspendus-tribunal-administratif-1665365.html > [Consulta: 15 de mayo de 2019]
} 
Les Vaîtes resultaba ser aquel lugar donde una comunidad diversa, sin necesidad de intervención municipal de ningún tipo, conseguía auto-organizarse en la autonomía más absoluta. Un intersticio para la vuelta a la tierra, en la ciudad, para un conocimiento creciente del suelo y sus comportamientos y para una convivencia transgeneracional y desclasada. Muchas personas jubiladas tenían huertos para autoconsumo y para entretenerse y enseñaban a las más jóvenes. Las técnicas de cultivo tradicional llegadas de diversos países dialogaban con la permacultura y a menudo una podía adivinar el origen de la persona que llevaba un huerto por las verduras que cultivaba.

Les Vaîtes era y es un lugar de memoria colectiva y de memoria migrante, un ecosistema donde surgían relaciones y vínculos con el conjunto de lo vivo, cooperando para vivir y cuidar juntas un algo común, donde la experiencia compartida agrandaba lo sensible, y donde cultivar se transformaba en cultivar los posibles (Macé, 2018).

\section{Tomar partido: el proyecto Nos jardins}

Marielle Macé habla de la necesidad de plantear la ecología en la actualidad, más allá del incremento de aprendizajes, conocimientos y nuevos dominios de cara a reparar o preservar el medio y sus habitantes. Nos invita a buscar y generar una filia, una amistad por la vida misma y por las diversas formas de vida existentes y el deseo de vincularse a ellas (Macé, 2018). Una filia, una empatía, un "formar parte de" un contexto en crisis y de una constelación de resistencias colectivas e individuales, que debería de llevarnos cada vez más y de forma urgente a asumir la imposibilidad de ser un solo individuo (Garcés, 2017) y empezar, desde las herramientas que son propias de cada una, a tomar la palabra y atreverse a pensar y actuar más allá de lo que una sabe (Garcés, 2018).

Tomar partido en un contexto como el de Les Vaîtes, siendo de fuera y estando apenas un mes era complejo. Sumarse a una lucha que no es la nuestra nunca es cosa fácil. Más allá del drama que suponía la pérdida de un espacio así para la ciudad y el colectivo, entraban en juego también pérdidas individuales, tierras por las que se había desarrollado un apego fuerte. En un contexto como éste ¿cómo no apropiarse indebidamente de un problema que no nos atraviesa directamente? ¿Qué relación deberíamos de mantener con el dolor ajeno? Marina Garcés, sumándose a un pensamiento de John Berger ${ }^{6}$ plantea que, aunque el dolor resulte incomunicable e intransferible, el deseo y la necesidad de responder a ese dolor sí son susceptibles de colectivizarse (Garcés, 2018).

De este modo, desarrollar un proyecto sobre Les Vaîtes, con las herramientas que me son propias (las de la producción artística), por muy humilde que fuera, me permitía sumarme a esa respuesta colectiva de rechazo utilizando los recursos que las circunstancias me ofrecían: medios físicos, algo de tiempo y una plataforma de difusión. Existía una (de tantas) necesidad de seguir difundiendo la problemática, llevarla fuera de la zona afectada y generar debates en torno a ella. En ese sentido, las prácticas artísticas tienen la capacidad de hacer visible lo que el consenso dominante suele oscurecer y borrar (Mouffe, 2007), aquello que el poder transforma en inexistente (Badiou, 2011), ya sea un movimiento de resistencia $u$ otros modos de vida. Pueden generar herramientas visuales que generan pequeñas rupturas, extrañamientos en el tejido de los sensible (Rancière, 2004) y socavar el marco imaginario necesario para la reproducción del sistema operante (Mouffe, 2007).

\footnotetext{
${ }^{6}$ Marina Garcés cita a John Berger en su libro Ciudad Princesa: "En esta edad oscura en la que vivimos, bajo el nuevo orden mundial, compartir el dolor es una de las condiciones previas esenciales para volver a encontrar la dignidad y la esperanza. Hay una gran parte del dolor que no puede compartirse. Pero el deseo de compartir dolor si puede compartirse. Y de esa acción, inevitablemente inadecuada, surge una resistencia." (Garcés, 2018, p. 136)
} 
Tras un mes de conversaciones y encuentros en Les Vaîtes, la sensación de infinitud de perfiles existentes allí me llevaba a la decisión de poner en primera fila lo que todos tenían en común: el espacio en sí. Aunque el contexto humano y las experiencias que generaba me parecían de lo más relevantes, la duración de mi estancia no me permitía lanzarme en un proyecto que hubiese implicado de cerca a otras personas. Implicar a otras personas hubiese requerido más tiempo para poder cuidar como toca de cada uno de los vínculos que se hubiesen generado a lo largo del proceso.
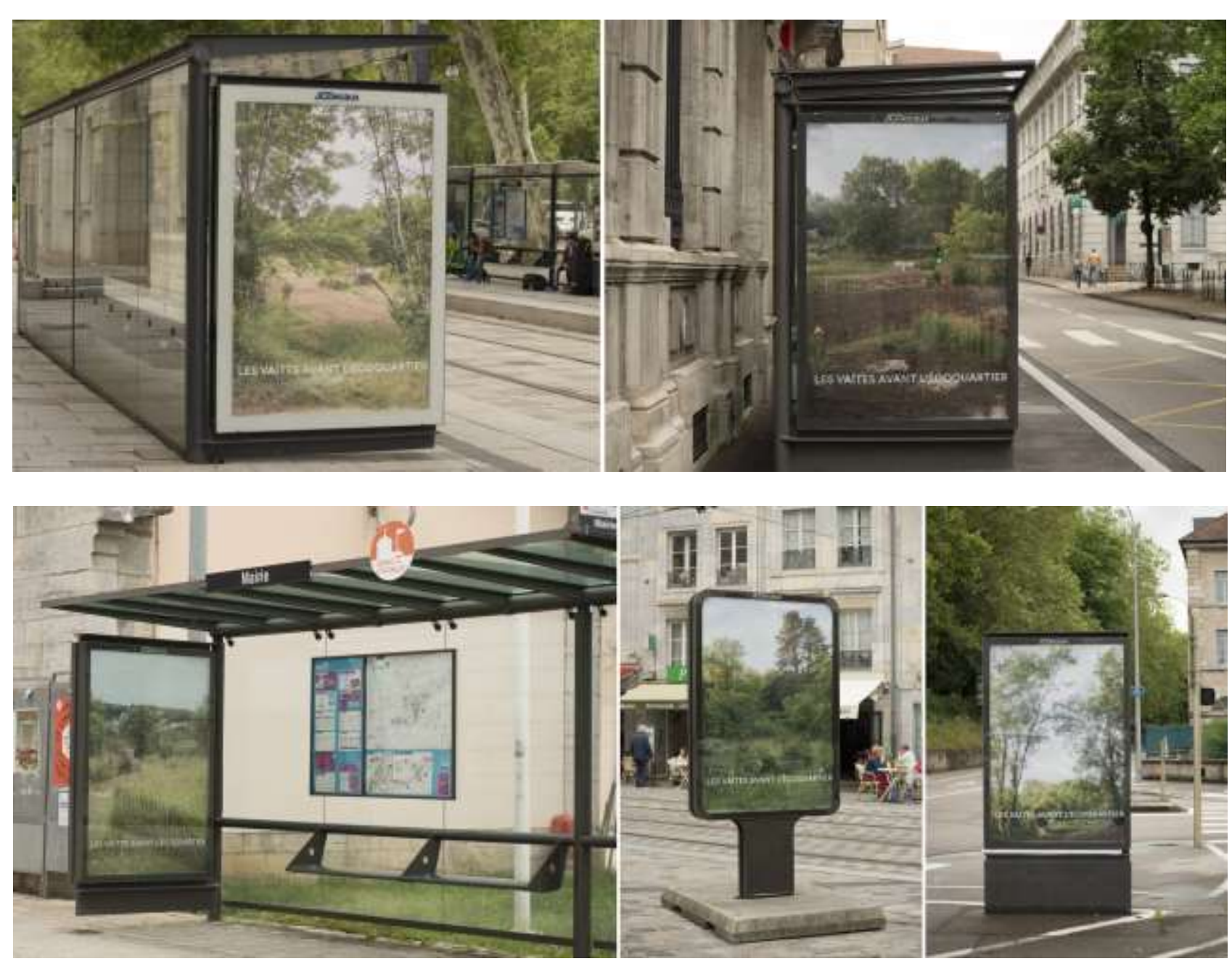

Fig. 2 y 3: Algunas de las intervenciones en marquesinas del proyecto Nos jardins.

Fotografía: Kristina Borhes, 2019.

El proyecto Nos jardins ${ }^{7}$ consistió así en dos tipos de intervenciones. La primera fue realizada en el centro de la ciudad y en la línea de tranvía que une el centro de la ciudad y el barrio de Les Vaîtes. Consistió en la realización de 24 carteles colocados en muppies y marquesinas de bus y de tranvía de JC Decaux, realizados a partir de fotografías tomadas durante los paseos llevados a cabo en Les Vaîtes. Sobre esas fotografías aparecía la frase Les Vaîtes avant l'écoquar$\operatorname{tier}^{8}$ (Les Vaîtes antes del ecobarrio) con el objetivo de facilitar la identificación de la zona para los viandantes, además de denunciar su futura e hipotética desaparición.

\footnotetext{
${ }^{7}$ Para ver más fotografías del proyecto: ANAIS FlORIN. Anaïs Florin <www.anaisflorin.com> [Consulta: 30 de agosto de 2019]

${ }^{8}$ El mensaje Les Vaîtes avant l'écoquartier (Les Vaîtes antes del ecobarrio) venía también a modo de guiño a aquellos pies de fotografía que encontramos a menudo en archivos de zonas que han desaparecido o se han visto fuertemente modificadas tras una guerra, una catástrofe natural o una reordenación del territorio.
} 
La segunda intervención tuvo lugar en Les Vaîtes, delante de la zona de huertos afectados por el proyecto urbanístico. Esta vez, la intervención consistía en el encolado de dos vallas publicitarias con algunos testimonios de personas que ocupaban los huertos con las que conversé durante mi estancia en Besançon.

"Toucher la terre c'est toujours un moment fort, c'est apaisant."

(Tocar la tierra siempre es un momento fuerte, es apaciguador)

"Ici on travaille avec le vivant."

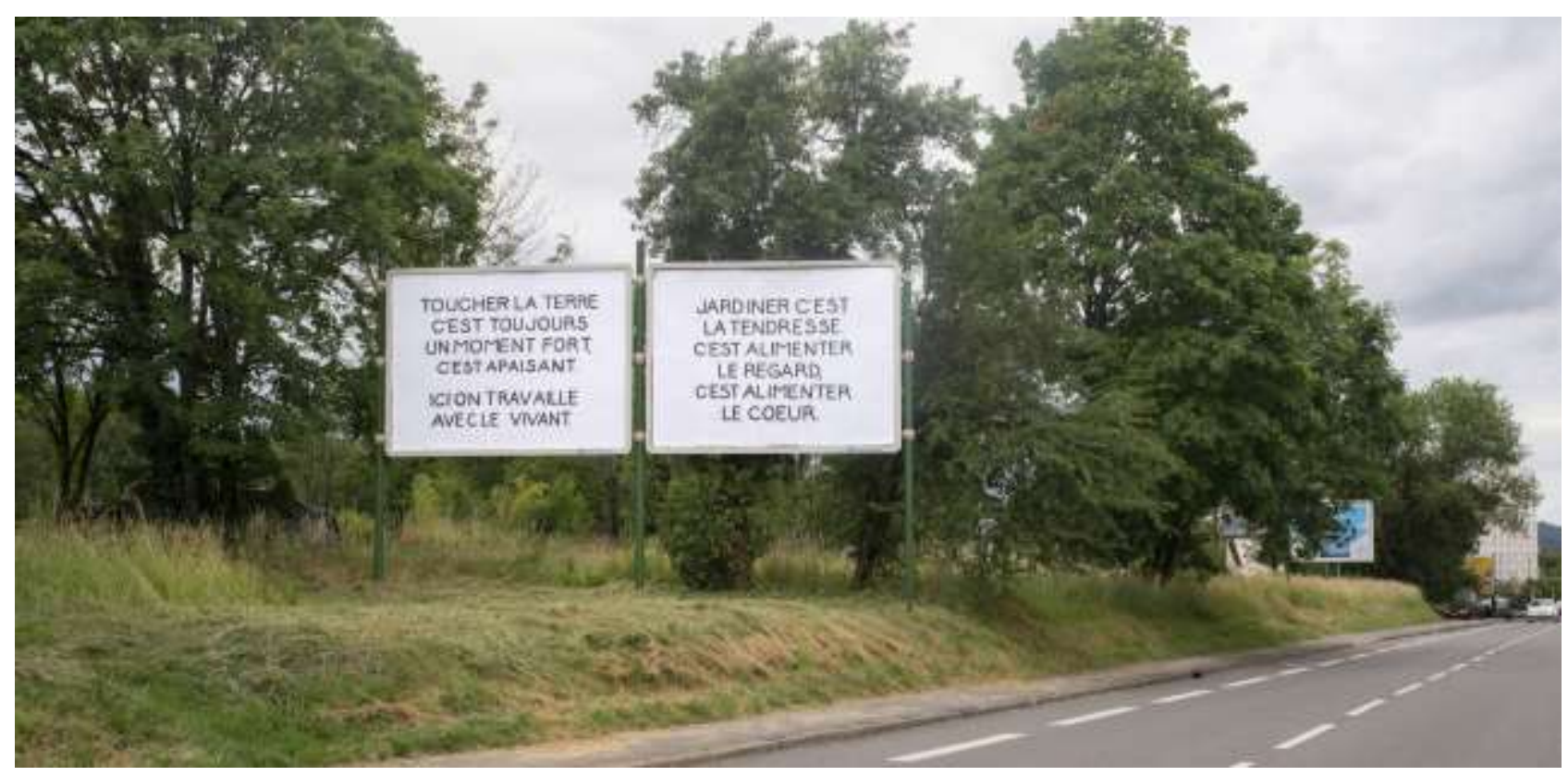

Fig. 4: Intervención en valla publicitaria del proyecto Nos jardins.

Fotografía: Anaïs Florin, 2019.

(Aquí trabajamos con la vida)

“Jardiner c'est la tendresse. C'est alimenter le regard, c'est alimenter le coeur."

(Cultivar es ternura. Es alimentar la mirada, alimentar el corazón.)

Las fotografías de la primera intervención hablaban de los espacios que podían desaparecer y la segunda se centraba sobre los lazos existentes en el territorio. Ambas intervenciones fueron realizadas de forma ilegal, cosa que el festival asumió sin ningún tipo de resistencia, aceptando servir de canal de difusión a la causa, dándole espacio en sus redes sociales y en su relación con los medios (se publicaron contenidos sobre la problemática y se concedieron entrevistas sobre las intervenciones). Tampoco hay que perder de vista que finalmente, Bien Urbain estaba financiando este proyecto, cosa que ponía al festival en una situación delicada ya que gran parte de su financiación venía de la municipalidad. Bien Urbain también tomaba partido.

Durante unos días las fotografías de las intervenciones se hicieron virales (a nivel local) en redes sociales y los medios de comunicación locales hablaron de ellas y de la temática que abordaban. La asociación Les jardins des Vaîtes las uti- 
lizó en varias ocasiones para difundir la problemática, y a ella se unieron plataformas y colectivos ecologistas, militantes, así como particulares que manifestaban su desaprobación al proyecto urbanístico. Ninguna duró más de una semana. Suficiente para hacer que algunas personas hablaran y que otras se enfadaran.

\section{A modo de conclusión}

Nos jardins es un proyecto que se enmarca en un contexto muy específico, una artista de fuera se asoma a una lucha ultra local desde el marco de un festival de arte público. Dadas las circunstancias, el proyecto dio lo máximo que podía dar, unas intervenciones, una visibilidad puntual de la problemática en el espacio público y un poco de difusión. No es mucho, pero es algo. No es comparable con estar en los huertos, ni con las meriendas o las visitas para conocer las especies protegidas locales que organizan las vecinas, pero sigue siendo algo. Evidentemente, este tipo de prácticas no cambia el curso de los eventos porque eso, como bien comenta Michel de Certeau, sería otorgarle un carácter mágico (De Certeau, 1994). No obstante, suma se intercala en las coordenadas de un mapa de posibles y puede a veces alterarlo, aunque sola sea un poco, como todas aquellas personas que forman parte de procesos de resistencia, cada una desde sus herramientas y sus vidas. Es importante acompañar, sumarse, construir lucha también en el imaginario colectivo.

Acercarse a un contexto en conflicto es acercarse a una comunidad específica herida y eso es algo a tener muy en cuenta en nuestras prácticas. Tenemos que aprender a acercarnos, a cuidar, a no alterar los tiempos que les son propios, a no cosificar y si las circunstancias lo exigen, retirarse, dar un paso atrás para no invadir el trabajo que ya desarrollan estas comunidades antes de nuestra llegada. A veces, creo que mucho del trabajo que nos queda por hacer desde este tipo prácticas tiene que ver con aprender que nuestras buenas intenciones son importantes para nosotras, pero no son tan relevantes dentro de un proceso colectivo que implica un dolor ajeno. Hay que sumarse pero no invadir, acompañar pero no abrumar. Cuidar las imágenes que generamos de aquellos colectivos, contrastarlas y cuestionarlas. Y para ello, es fundamental el factor tiempo. Trabajar durante un mes sobre un contexto nuevo como fue el caso de Nos jardins es una temporalidad realmente corta que no permite establecer vínculos reales y que requiere de un cuidado extremo para no generar desencuentros. De hecho, yo no lo conseguí. Aunque el retorno general sobre el proyecto fue muy positivo, una persona con la que había trabajado no se sentía representada por las intervenciones. Me dijeron que no me preocupara, que no podía gustarle a todo el mundo, y es verdad. Pero también es verdad que no hay que perder de vista estas experiencias y revisar cómo podría haber gestionado esta situación mejor, y a menudo, el factor tiempo es crucial para hacerlo. Existe una necesidad real de revisar nuestras metodologías a lo hora de trabajar en contexto y no dejar nunca de ser honestas y críticas con nosotras mismas.

Y es importante repensarnos y trabajar, porque es fundamental proteger lugares como Les Vaîtes, por lo que son desde un punto de vista medioambiental y por lo que representan desde el punto de vista humano, emocional y político. Hay que cuidar de los espacios de autonomía y sumarnos a su cultivo. Tenemos que generar herramientas nuevas desde todas nuestras disciplinas para tender a construir un nosotros fuerte y plural. Un nosotros para cuidarnos, acompañarnos, apoyarnos, ser cómplices en la lucha y en la vida. Y hay que elegir si estar con nosotros o con ellos, hay que tomar partido.

\section{Referencias}

ASSOCIATION LES JARDINS DES VAITES. Les jardins des Vaîtes < https://www.facebook.com/jardinsdesvaites/> [Consulta: el 30 de agosto de 2019]

Association JUSTE ICI. Bien Urbain, Art dans et avec l'espace public <https://bien-urbain.fr> [Consulta: el 30 de agosto de 2019] BADIOU, A.. (2011) Le réveil de l'histoire. Paris: Nouvelles Éditions Lignes 
Tomar partido: práctica artística y visibilización de conflictos, el caso del proyecto "Nos Jardins".

COURAGEOT, S. (2019) “Besançon : Les travaux du futur éco-quartier des Vaîtes suspendus par le tribunal administratif.”, 5 de mayo. <https://france3-regions.francetvinfo.fr/bourgogne-franche-comte/doubs/besancon/besancon-travaux-du-futur-eco-quartier-vaitessuspendus-tribunal-administratif-1665365.html $>$ [Consulta: el 15 de mayo de 2019]

DE CERTEAU, M.. (1994) La prise de parole et autres écrits politiques. Paris: Éditions du Seuil.

FLORIN, A.Anaïs Florin <www.anaisflorin.com> [Consulta: el 30 de agosto de 2019]

GARCÉS, M. (2017) Nueva ilustración radical. Barcelona: Nuevos Cuadernos Anagrama.

GARCÉS, M.(2018) Ciudad Princesa. Barcelona: Galaxia Gutenberg.

GARCÉs, M.(2013) Un mundo común. Barcelona: Edicions Bellaterra.

MACÉ, M. (2019) Nos cabanes. Paris: Éditions Verdier.

MOUfFe, C. (2007) Prácticas artísticas y democracia agonística. Barcelona: Museu d'Art Contemporani de Barcelona.

RANCIERE, J. (2004) Malaise dans l'esthetique. Paris: Editions Galilée.

TERRITOIRE 25. L'écoquartier Les Vaîtes 〈http://www.les-vaites.fr/> [Consulta: el 30 de agosto de 2019] 